\title{
El género de los protagonistas en la información deportiva (1979-2010): noticias y titulares
}

\author{
Clara SAINZ DE BARANDA ANDÚJAR \\ Universidad Carlos III de Madrid \\ cbaranda@hum.uc3m.es
}

Recibido: 7 de octubre de 2013

Aceptado: 21 de abril de 2014

\begin{abstract}
Resumen
La investigación que se presenta tiene como objeto analizar la información deportiva dentro de una triple realidad: espacial (España), temporal (1979-2010) y de género. Mediante un muestreo aleatorio, se analizaron un total de 95.439 informaciones publicadas durante una semana desde el año 1979 hasta el año 2010 en los cuatro diarios deportivos españoles de mayor tirada. La mujer es sujeto noticioso únicamente en el $5,11 \%$ de los casos -unas veces sola $(2,18 \%)$ y otras acompañada por al menos un protagonista masculino (2,93\%)-, mientras que el hombre lo es en el 92,24\%. Se mantienen los mismos porcentajes con el paso de los años. Los resultados confirman cómo los diarios especializados deportivos han aumentado la invisibilidad de las mujeres a pesar de los logros deportivos protagonizados en los últimos años.
\end{abstract}

Palabras clave: prensa deportiva, género, deporte, titulares.

\section{The gender of the main characters in sports reporting (1979-2010): News and headlines}

\begin{abstract}
The research presented in this paper is to analyze sport information in a triple reality: Spatial (Spain), seasonal (1979-2010) and gender. By random sampling, analyzed a total of 95.439 information published for a week from 1979 to 2010 in the four Spanish sports newspapers with the largest circulation. The woman is subjected news only $5.11 \%$ of cases -sometimes alone $(2.18 \%)$ and sometimes with at least one male partner $(2.93 \%)$ - at the same time males is in $92.24 \%$ of the cases. It remains the same percentages with the passage of years. The results confirm how newspapers have specialized sports increased the invisibility of women despite the achievements featuring sports in recent years.
\end{abstract}

Keywords: sports news, gender, sport, headlines.

\section{Referencia normalizada}

SAINZ DE BARANDA ANDÚJAR, Clara (2014): "El género de los protagonistas en la información deportiva (1979-2010): noticias y titulares". Estudios sobre el Mensaje Periodístico. Vol. 20, Núm. 2 (julio-diciembre), págs.: 1225-1236. Madrid, Servicio de Publicaciones de la Universidad Complutense.

Sumario:1. Introducción. 2. Metodología; 2.1. Muestra; 2.2. Instrumento y procedimiento. 3. Resultados; 3.1. Género de los protagonistas; 3.2.1. Nombres propios en los títulos; 3.2.2. Citas en los títulos. 4. Discusión. 5. Conclusiones. 6. Referencias bibliográficas.

\section{Introducción}

En los Juegos de la XXX Olimpíada en Londres, las mujeres compitieron en todos los deportes incluidos en el programa. Un 44\% de los 10.875 deportistas inscritos eran mujeres, en el caso de Estados Unidos las mujeres superaron en número a sus compañeros hombres, y todos los Comités Olímpicos Nacionales enviaron mujeres (COI). Por primera vez las deportistas españolas han obtenido mejores resultados que los de- 
portistas en los últimos Juegos Olímpicos de Londres 2012. El deporte femenino en el siglo XX ha dejado de ser anecdótico'.

La información deportiva tiene una gran demanda en la sociedad actual: Marca es el diario de pago más leído en España, con casi tres millones de lectores al día; $A s$ ocupa el tercer puesto; y Mundo Deportivo y Sport el sexto y séptimo puesto respectivamente (EGM, 2012). Además, en los últimos años se ha producido un aumento del número de lectores en prensa diaria deportiva, cosa que no ocurre con los diarios generalistas.

Estos datos definen un escenario en el que la prensa deportiva, hoy en día, es un medio especializado -impreso o digital-, cualquiera que sea su periodicidad, que se dirige a una audiencia generalista identificada con el mayor seguimiento a unos determinados equipos o actividades deportivas.

Los medios de comunicación constituyen una ineludible fuente de información e influencia para la ciudadanía (Koivula, 1999) por lo que se hace necesario revisar cual es tratamiento de la información deportiva en general con la imagen que estos medios transmiten de las mujeres.

Algunos trabajos en España observan como en los medios generalistas las mujeres aparecen en un segundo orden, como protagonistas y como fuentes, entre tanto los hombres son protagonistas principales de las informaciones.

El objetivo es analizar a la mujer deportista en un contexto de superación del viejo paradigma, en el que, a lo largo del siglo XX, ha conseguido la consideración de deportista, o sea con plenos derechos a la actividad física, en tanto que competidoras, y no como meras observadoras o como excepción anecdótica. La visión longitudinal del presente estudio (1979-2010) permite observar la evolución -o el inmovilismo- de los contenidos deportivos y de la imagen que de las mujeres proyecta la prensa deportiva.

\section{Metodología}

\subsection{Muestra}

Para el estudio observacional de la prensa escrita, se ha utilizado la metodología observacional propuesta por Anguera (2011).

Mediante un muestreo aleatorio, se analizaron todas las informaciones de prensa deportiva de los periódicos Marca, As, Mundo Deportivo y Sport, que se publicaron durante una semana desde el año 1979 hasta el año 2010. Se analizaron, por tanto, un total de 4 periódicos x 32 semanas $x 7$ días, es decir 896 periódicos, con un total de 95.439 informaciones. Para ello, y siguiendo la propuesta de Pérez (2009), se escogió mediante un muestreo aleatorio simple un número de semana al año (52 semanas al año, 56 en los años bisiestos) y un día de la semana, registrando todas las informaciones desde ese día de la semana hasta el día anterior de la semana siguiente².

1 Si tenemos en cuenta que no se superó el 10\% de participación femenina hasta los Juegos de Seúl 1984, que en Sydney 2000 el 38\% de los 10.382 atletas inscriptos eran mujeres y que estas compitieron en 25 de los 28 deportes y que, a escala internacional no se ha conseguido que todos los países envíen mujeres representándolos hasta el año 2012, el avance es considerable.

2 Aquellos días comprendidos en el período de análisis en los que no se ha podido localizar la fuente primaria para su análisis se opta por analizar el diario del día consecutivo. 


\subsection{Instrumento y procedimiento}

El instrumento utilizado para el análisis de las informaciones fue "la guía de análisis de prensa deportiva" propuesta por Sainz de Baranda (2013) al proporcionar unos índices de fiabilidad y validez de contenido muy altos (Kappa $<0.94$, en el peor de los casos).

Las variables objeto de estudio fueron: la fecha de publicación; el género de las personas que aparecen en la información, sea cual sea su papel -protagonista principal o secundario de la información, fuente o protagonista de la fotografía si la hubiera (femenino, masculino, 'mixto' y 'neutro'); y en los titulares la inclusión de acto de habla en el título y la inclusión de un nombre propio en el título.

Atendiendo a las características propias del estudio se ha optado por analizar las siguientes variables: de la clasificación, detallada, propuesta por López Hidalgo (2001: 49-56) de los títulos periodísticos en siete modalidades, interesan únicamente de los denominados títulos de actos de habla o títulos declarativos. López Hidalgo propone tres procedimientos de cita en los títulos: la cita directa, la indirecta y la mixta, donde se combina la directa e indirecta. En este sentido, en el presente estudio interesa conocer la tendencia existente en la prensa deportiva de "la situación comunicativa predominante en la información periodística consiste en que alguien informa sobre lo dicho por alguien" (Hurtado, 2009: 192) y como se representa en los títulos.

La segunda variable a analizar es la inclusión de un nombre propio en el título, se ha optado indicar, en el caso que el título incluya un nombre propio, si el nombre es un nombre propio de persona (femenino, masculino, o ambos) o de un equipo (femenino, masculino, o ambos).

El análisis fue realizado por un observador. Para la formación en el contexto de la observación se realizaron cuatro sesiones de dos horas de observación, en el que se registraron dos ejemplares de cada uno de los cuatro diarios objeto de estudio, escogidos al azar, pertenecientes a la población pero no a la muestra final. Trascurrido una semana, y en circunstancias prácticamente idénticas al anterior registro, se volvió a registrar los mismos ejemplares analizados anteriormente. Se observaron índices de fiabilidad muy altos (Kappa $=0.94$, en el peor de los casos).

Con el objetivo de asegurar el buen comportamiento del observador, y evitar que durante el proceso de toma de datos se arrastrase un error, o hubiese pequeñas modificaciones de criterio, se decidió realizar una última prueba, posterior a la recogida de datos. Esta prueba de fiabilidad consistió en volver a analizar un ejemplar de cada uno de los cuatro diarios de pago objeto de estudio, analizados en el proceso de entrenamiento del observador. Se observaron de nuevo índices de fiabilidad muy altos (Kappa $=0.95$, en el peor de los casos).

Para el análisis de los resultados se realizó un análisis descriptivo e inferencial de los datos (Test de Chi-cuadrado y tablas de contingencia) con un nivel de significación estadística de $\mathrm{p}<.05$. El análisis estadístico de los datos fue calculado con el programa Spssv.19.0.

\section{Resultados}

El género como categoría de análisis nos remite a una noción de relación: relación de las mujeres con su entorno, pero también como contraposición a los hombres. Se ex- 
ponen los resultados de la evolución de los protagonistas de las informaciones de la prensa deportiva según género y de los títulos de las mismas, teniendo en cuenta el total de la muestra $(\mathrm{N}=95.439)$.

Se trata de mostrar el panorama general de la prensa deportiva desde la perspectiva de género y por lo tanto, abordar el papel de las mujeres desde una posición de unión, no aisladas en un mundo sin ningún tipo de vínculos con el exterior. Ese papel que las mujeres desempeñan en el nuevo entorno social no puede entenderse en un mundo segregado del de los hombres. Ambos son parte integrante de un mismo sistema, de una misma realidad. Una realidad en la que los medios de comunicación se convierten en transmisores de los valores y esquemas presentes en la sociedad, pero también en generadores de nuevas formas de relación.

\subsection{Género de los protagonistas}

Los hombres son los protagonistas indiscutibles de la prensa deportiva. Los resultados de la investigación muestran que las mujeres siguen estando infrarrepresentadas en las páginas de los diarios deportivos españoles, es más, a lo largo del tiempo disminuyen su presencia.

El porcentaje del total de informaciones según el género de los protagonistas en los diarios deportivos describe (tabla 1) que en un 92,24\% las informaciones las protagonizan hombres. Únicamente en el $5,11 \%$ de los casos las mujeres son sujeto noticioso, unas veces sola $(2,18 \%)$ y otras acompañada por al menos un protagonista masculino (2,93\%), codificadas como 'mixtas'. En un 2,65\% las informaciones las protagoniza una institución, ley, normativa, competición, instalaciones, medios de comunicación, etc., codificadas como 'neutras'.

Si nos centramos en la evolución ${ }^{3}$ de las informaciones según el género de los sujetos noticiosos, los datos de la tabla 1 señalan que los hombres mantienen su hegemonía como sujeto noticioso en la prensa deportiva española durante los últimos 32 años, sólo en el período comprendido entre los años 1980 y 1984 se contempla un pequeño descenso, por primera y única vez, por debajo del 90\%. Durante los años siguientes aumenta y llega a superar el $94 \%$ de las informaciones totales.

La mujer como sujeto noticioso se sitúa en torno al $2 \%$ en los años analizados. En la primera década del siglo XXI permanece por debajo del 2\%, observándose así un descenso de la información sobre mujer en la prensa deportiva. En el año 2010 se recupera y vuelve a estar por encima del $2 \%$.

Hombre y mujer comparten protagonismo en el 2,04\% de las informaciones analizadas en el año 1979. Durante la década de los ochenta aumenta llegando a situarse dos puntos por encima; sin embargo, en los noventa comienza a disminuir la información 'mixta' paulatinamente y llega a situarse por debajo del $2 \%$ en la primera década del siglo XXI. En el año 2010 la información 'mixta' supone un 2,01\%.

3 Durante la exposición de las tablas de evolución se opta por mostrar los datos íntegros del primer y último año y agrupar los años centrales en lustros para facilitar la descripción de la muestra. 
En 1979, al analizar las informaciones con protagonistas 'neutros' suponen un 3\%. Durante la década de los ochenta la información 'neutra' aumenta (más de medio punto), pero en los años noventa comienza a disminuir y llega a situarse en torno al $2 \%$ en el año 2010.

Tabla 1. Evolución de las informaciones de la prensa deportiva según el género de los protagonistas

\begin{tabular}{|l|r|r|r|r|r|r|r|r|r|}
\hline & $\mathbf{1 9 7 9}$ & $\mathbf{1 9 8 0}$ & $\mathbf{1 9 8 5}$ & $\mathbf{1 9 9 0}$ & $\mathbf{1 9 9 5}$ & $\mathbf{2 0 0 0}$ & $\mathbf{2 0 0 5}$ & \multirow{2}{*}{$\mathbf{1 9 1 0}$} & TOTAL \\
\hline Hombres & \multicolumn{1}{|c|}{92,65} & 89,85 & 90,25 & 90,8 & 92,46 & 94,20 & 94,23 & 93,59 & 92,24 \\
\hline Mujeres & 2,32 & 2,08 & 2,54 & 2,46 & 2,45 & 1,62 & 1,96 & 2,37 & 2,18 \\
\hline 'Mixtas' & 2,04 & 4,13 & 3,8 & 3,93 & 2,87 & 1,87 & 1,96 & 2,01 & 2,93 \\
\hline 'Neutras' & 3,00 & 3,93 & 3,41 & 2,81 & 2,21 & 2,31 & 1,85 & 2,03 & 2,65 \\
\hline
\end{tabular}

Los datos de la tabla 1 indican relaciones estadísticamente significativas entre las variables género del protagonista y la evolución temporal $(\chi 2(21, \mathrm{~N}=95439)=529,173, \mathrm{p}=.001)$.

\subsection{Los titulares}

Los titulares en los diarios han tenido tradicionalmente tres funciones: la propiamente informativa, la de atraer al lector a su lectura y la de identificar y diferenciar esa información de otras. En esta última función nos centramos a la hora de analizar la identificación de los protagonistas en el título -con el que el lector tiene su primer acercamiento- con sus nombres propios, de persona o de equipos, y sus voces, las citas, dos características frecuentes en los títulos de las informaciones. Los datos analizados confirman que en la prensa deportiva la inclusión de un nombre propios $(65,88 \%)$ en los títulos periodísticos es más frecuente que la inclusión de citas $(8,26 \%)$.

\subsubsection{Nombres propios en los títulos}

El total de los títulos que incluyen nombres propios evolucionan a lo largo del tiempo (tabla 2) de manera ascendente. Al comparar el primer período, 1980-1984 (61,01\%), y el último, 2005-2009 (67,31\%), asciende más de siete puntos. Sin embargo, al comparar el año $1979(61,77 \%)$ y el año $2010(65,88 \%)$ el ascenso es menor. Por lo que se puede decir que existe una tendencia ascendente hasta principios del siglo XXI en el que se inicia un retroceso vigente en el último año analizado.

Tabla 2. Evolución de la inclusión de nombres propios en los títulos periodísticos según el género

\begin{tabular}{|l|r|r|r|r|r|r|r|r|r|}
\hline $\begin{array}{l}\text { NOMBRES EN } \\
\text { LOS TÍTULOS }\end{array}$ & $\mathbf{1 9 7 9}$ & $\begin{array}{l}\mathbf{1 9 8 0} \\
\mathbf{1 9 8 4}\end{array}$ & $\begin{array}{l}\mathbf{1 9 8 5 -} \\
\mathbf{1 9 8 9}\end{array}$ & $\begin{array}{l}\mathbf{1 9 9 0} \\
\mathbf{1 9 9 4}\end{array}$ & $\begin{array}{l}\mathbf{1 9 9 5 -} \\
\mathbf{1 9 9 9}\end{array}$ & $\begin{array}{l}\mathbf{2 0 0 0 -} \\
\mathbf{2 0 0 4}\end{array}$ & $\begin{array}{l}\mathbf{2 0 0 5 -} \\
\mathbf{2 0 0 9}\end{array}$ & $\mathbf{2 0 1 0}$ & TOTAL \\
\hline Persona Masculino & 36,32 & 34,27 & 38,97 & 44,25 & 42 & 41,87 & 43,32 & 40,05 & 40,90 \\
\hline Equipo Masculino & 23,29 & 24,34 & 21,93 & 21,02 & 20,95 & 22,98 & 24,13 & 25,3 & 22,69 \\
\hline Persona Femenino & 0,72 & 1,23 & 1,97 & 2,16 & 1,8 & 1,04 & 1,4 & 1,32 & 1,55 \\
\hline Equipo Femenino & 1,24 & 0,75 & 0,58 & 0,43 & 0,32 & 0,28 & 0,28 & 0,37 & 0,44 \\
\hline $\begin{array}{l}\text { Persona Femenino } \\
\text { y Masculino }\end{array}$ & 0,20 & 0,34 & 0,26 & 0,22 & 0,23 & 0,23 & 0,22 & 0,18 & 0,24 \\
\hline $\begin{array}{l}\text { Equipo Femenino y } \\
\text { Masculino }\end{array}$ & 0,00 & 0,06 & 0,08 & 0,03 & 0,04 & 0,02 & 0,04 & 0,08 & 0,04 \\
\hline Total nombres & 61,77 & 61,01 & 63,80 & 68,10 & 65,35 & 66,41 & 69,39 & 67,31 & 65,88 \\
\hline No hay nombres & 38,23 & 38,99 & 36,2 & 31,9 & 34,65 & 33,59 & 30,61 & 32,69 & 34,12 \\
\hline
\end{tabular}

Los datos de la tabla 2 indican relaciones estadísticamente significativas entre las variables nombres propios en los títulos y la evolución temporal $(\chi 2(7, \mathrm{~N}=95439)=309,487, \mathrm{p}=.001)$. 
$\mathrm{Al}$ analizar de manera pormenorizada la inclusión un nombre propio según el género (tabla 2) se observa que el nombre de persona masculino es el que más abunda $(40,90 \%)$, seguido del de equipo masculino $(22,69 \%)$, el de persona femenino $(1,55 \%)$, el de equipo femenino $(0,44 \%)$, cuando comparten título el nombre persona femenino y masculino $(0,24 \%)$ y por último cuando comparten título el nombre de equipo femenino y masculino $(0,04 \%)$.

En este sentido, cuando hablamos de evolución, los títulos que incluyen un nombre de persona masculino evolucionan a lo largo del tiempo de manera ascendente: al comparar el primer período, 1980-1984 (34,27\%), y el último, 2005-2009 (43,32\%), asciende más de nueve puntos; a finales de los ochenta los títulos con un nombre de persona masculino superan a los que en ningún caso incluyen nombre propio, pero es en el período comprendido entre 1990-1994 cuando se produce la frecuencia más alta $(44,25 \%)$.

En gran medida el aumento del nombre de persona masculino viene condicionado por el descenso de los títulos que no incluyen nombres. Al comparar el primer período, 1980-1984 (38,99\%), y el último, 2005-2009 (30,61\%), desciende más de ocho puntos.

Los títulos que incluyen nombre de equipo masculino mantienen su frecuencia por encima del $20 \%$ durante todos los períodos analizados. En el año 2010 suponen un $25,3 \%$, su frecuencia más alta.

Los títulos que incluyen nombre de persona femenino evolucionan de manera ascendente hasta el periodo 1990-1994, en el que marcan su frecuencia más alta (2,16\%). A partir de ese momento descienden y en el año 2010 suponen tan solo un 1,32\%.

Los nombres femeninos de equipo y de persona y los de equipo que incluyen en el mismo título femenino y masculino, mantienen frecuencias muy bajas durante todo el análisis, por debajo del 1\%. En este sentido, parece que los equipos femeninos no interesan a la prensa deportiva.

\subsubsection{Citas en los títulos}

En general, las citas en los títulos son poco frecuentes (tabla 3). El porcentaje del total de citas en los títulos es de un 8,26\%: de los que un 7,85\% lo hace en forma de cita textual, un $0,37 \%$ en forma de cita indirecta y un $0,04 \%$ combina cita directa e indirecta.

En la tabla 3 se observa baja frecuencia e inconstancia en el tiempo de los títulos que incluyen citas indirectas y los títulos en el que se combinan las citas directas e indirectas, de tal modo que a la hora de describir los resultados se tienen en cuenta los datos de forma agrupada, teniendo en cuenta las citas totales que aparecen en los títulos.

Tabla 3. Evolución de la inclusión de citas en los títulos periodísticos

\begin{tabular}{|l|r|r|r|r|r|r|r|r|r|}
\hline $\begin{array}{l}\text { CITAS EN LOS } \\
\text { TÍTULOS }\end{array}$ & $\mathbf{1 9 7 9}$ & $\begin{array}{l}\mathbf{1 9 8 0} \\
\mathbf{1 9 8 4}\end{array}$ & $\begin{array}{l}\mathbf{1 9 8 5} \\
\mathbf{1 9 8 9}\end{array}$ & $\begin{array}{l}\mathbf{1 9 9 0} \\
\mathbf{1 9 9 4}\end{array}$ & $\begin{array}{l}\mathbf{1 9 9 5} \\
\mathbf{1 9 9 9}\end{array}$ & $\begin{array}{l}\mathbf{2 0 0 0} \\
\mathbf{2 0 0 4}\end{array}$ & $\begin{array}{l}\mathbf{2 0 0 5} \\
\mathbf{2 0 0 9}\end{array}$ & $\mathbf{2 0 1 0}$ & Total \\
\hline $\begin{array}{l}\text { En forma de cita } \\
\text { textual }\end{array}$ & 7,27 & 6,07 & 7,35 & 9,69 & 8,72 & 6,90 & 8,08 & 8,15 & 7,85 \\
\hline $\begin{array}{l}\text { En forma de cita } \\
\text { indirecta }\end{array}$ & 0,12 & 0,32 & 0,23 & 0,42 & 0,53 & 0,41 & 0,26 & 0,53 & 0,37 \\
\hline $\begin{array}{l}\text { Mixtos de cita directa e } \\
\text { indirecta }\end{array}$ & 0,00 & 0,01 & 0,02 & 0,04 & 0,06 & 0,07 & 0,05 & 0,08 & 0,04 \\
\hline Total citas & 7,39 & 6,40 & 7,60 & 10,15 & 9,31 & 7,38 & 8,39 & 8,76 & 8,26 \\
\hline No incluye cita & 92,61 & 93,60 & 92,4 & 89,85 & 90,69 & 92,62 & 91,62 & 91,24 & 91,74 \\
\hline
\end{tabular}


En general la inclusión de citas en los títulos aumenta hasta los años noventa, cuando se producen las frecuencias más altas (un 10,15\% a principios de la década y un 9,31\% a finales). En el siglo XXI desciende la frecuencia, aunque su frecuencia es mayor que en los primeros años del análisis.

Los datos de la tabla 3 indican relaciones estadísticamente significativas entre las variables citas en los títulos y la evolución temporal $\left(\chi^{2}(7, \mathrm{~N}=95439)=174,675\right.$, $\mathrm{p}=.001$ ).

En la tabla 4 se observa la inclusión de citas en los títulos periodísticos según el género de los protagonistas. Se observa que los títulos de las informaciones en las que los hombres son protagonistas incluyen citas en un $8,78 \%$ : de los que un $8,35 \%$ son citas textuales, un $0,39 \%$ son indirectas y en un $0,05 \%$ se combinan la cita directa e indirecta.

En el caso de los títulos de las informaciones protagonizadas por mujeres incluyen en un 3,07\% de sus títulos citas: de los que un $2,98 \%$ son citas directas, un $0,1 \%$ son indirectas y en ningún caso se combinan la cita directa e indirecta.

En el caso de los títulos de las informaciones 'mixtas' incluyen en un 3,08\% de sus títulos citas: de los que un $2,93 \%$ son citas textuales, un $0,14 \%$ son indirectas y en ningún caso se combinan la cita directa e indirecta.

Tabla 4. Inclusión de citas en los títulos periodísticos según el género de los protagonistas

\begin{tabular}{|c|c|c|c|c|c|c|c|c|c|}
\hline & \multicolumn{2}{|c|}{ Hombre } & \multicolumn{2}{|c|}{ Mujer } & \multicolumn{2}{|c|}{ 'Mixto' } & \multicolumn{2}{|c|}{ 'Neutro' } & \multirow{2}{*}{$\begin{array}{c}\text { Total } \\
\% \text { del } \mathrm{N} \\
\text { de la } \\
\text { columna }\end{array}$} \\
\hline $\begin{array}{l}\text { EN LOS } \\
\text { TÍTULOS }\end{array}$ & $\begin{array}{c}\% \text { del } N \\
\text { de la } \\
\text { columna }\end{array}$ & $\begin{array}{l}\% \text { de } \\
\text { la fila }\end{array}$ & $\begin{array}{l}\% \text { del } \mathrm{N} \\
\text { de la } \\
\text { columna }\end{array}$ & $\begin{array}{c}\% \\
\text { de la } \\
\text { fila }\end{array}$ & $\begin{array}{c}\% \text { del } \mathrm{N} \\
\text { de la } \\
\text { columna }\end{array}$ & $\begin{array}{c}\% \text { de } \\
\text { la } \\
\text { fila }\end{array}$ & $\begin{array}{c}\% \text { del } \mathrm{N} \\
\text { de la } \\
\text { columna }\end{array}$ & $\begin{array}{c}\% \text { de } \\
\text { la } \\
\text { fila }\end{array}$ & \\
\hline $\begin{array}{l}\text { Incluye } \\
\text { citas }\end{array}$ & 8,78 & 98,10 & 3,07 & 0,81 & 3,08 & 1,09 & 0 & 0 & 8,26 \\
\hline $\begin{array}{l}\text { No incluye } \\
\text { citas }\end{array}$ & 91,22 & 91,71 & 96,93 & 2,30 & 96,92 & 3,09 & 100 & 2,89 & 91,74 \\
\hline Total & 100 & 92,24 & 100 & 2,18 & 100 & 2,93 & 100 & 2,65 & 100 \\
\hline
\end{tabular}

Al analizar la tabla 4 en función del género queda claro que el 98,1\% de los títulos que incluyen citas son de informaciones protagonizadas por hombres, solo el $0,81 \%$ las protagonizan mujeres y el $1,09 \%$ son 'mixtas'.

Los datos de la tabla 4 indican relaciones estadísticamente significativas entre las variables género del protagonista y citas en los títulos $\left(\chi^{2}(3, \mathrm{~N}=95439)=432,547\right.$, $\mathrm{p}=.001)$.

\section{Discusión}

A pesar de los progresos que el deporte femenino ha hecho a lo largo del siglo XX y a los resultados obtenidos por las deportistas en los últimos años, la equiparación con respecto a los hombres es lenta en relación a la representación que los medios de comunicación hacen de los logros y avances de las mujeres deportistas.

Un buen ejemplo es que apenas 91 centésimas de diferencia, es lo que separa el récord mundial masculino en 100 metros lisos (Usain Bolt) del femenino (Florence Griffith Joyner), mientras que una desigualdad de más de noventa puntos es lo que separa a la representación de la mujer como sujeto noticioso del hombre. 
Las mujeres siguen estando infrarrepresentadas en las páginas de los diarios deportivos españoles más demandados por el público. De hecho, el mayor hallazgo, en la línea de lo que anunciaban los datos del Global Media Monitoring Project (GMMP) (WACC, 2010) para España -en medios de comunicación generalistas-, es el retroceso que se ha producido en las informaciones protagonizadas por mujeres en los cuatro diarios deportivos españoles analizados entre los años 1979 y 2010.

Queda claro que la información protagonizada por hombres se incrementa a lo largo del tiempo en detrimento del resto. De hecho, si se comparan estos datos con los ofrecidos por otras investigaciones (tabla 5), se observa que la invisibilidad de las mujeres en la prensa deportiva española, además de aumentar a lo largo del tiempo, es mayor que en las informaciones deportivas registradas en otros medios y otros países.

En el total de las informaciones, existen relaciones estadísticamente significativas entre las variables género del protagonista y evolución temporal. De hecho, los datos generales muestran que en el año 2010 la frecuencia de las mujeres como protagonistas de las informaciones, es igual (tabla 1, apenas unas centésimas de diferencia) que la obtenida para el año 1979.

En 1979 las informaciones con protagonistas femeninas -las de mujeres (2,32\%) y 'mixtas' (2\%)- suponen un $4,32 \%$ del total. A principios de los años ochenta los datos reflejan un aumento de la información protagonizada por mujeres, cuando Evert y Navratilova disputaban su corona en el tenis femenino, el deporte "reina", y que tímidamente aumenta a finales de esta misma década con nuevas protagonistas, recuérdese a Steffi Graf. En años posteriores la realidad mediática muestra que fue sólo un espejismo.

El 10 de junio de 1989, Arancha Sánchez Vicario, una joven desconocida de 17 años, derrotaba en la final de Roland Garros a la todopoderosa Steffi Graf. Comienza una nueva etapa, en la que Arancha Sánchez Vicario -la mejor tenista española de todos los tiempos, número uno mundial en 1995- y Conchita Martínez -de manera casi paralela, número dos en 1996- acrecientan la fama española a nivel mundial. Las primeras medallas para las deportistas femeninas españolas en unos Juegos Olímpicos se hacen realidad en 1992.

Tabla 5. Revisión de la investigación académica: porcentaje de informaciones deportivas en medios generalistas en relación al género (Elaboración propia)

\begin{tabular}{|l|l|l|c|c|c|c|}
\hline \multicolumn{1}{|c|}{ Autores } & \multicolumn{1}{c|}{ País } & \multicolumn{1}{c|}{ Medio } & Mujeres & Hombres & Ambos & Neutro \\
\hline Duncan y cols. (1989) & EE.UU. & TV & $5,0 \%$ & $92,0 \%$ & - & $3,0 \%$ \\
\hline Duncan y cols. (1991) & EE.UU. & Periódico & $3,5 \%$ & $81,5 \%$ & $12,1 \%$ & $3,4 \%$ \\
\hline Duncan y cols. (1994) & EE.UU. & TV & $5,0 \%$ & $94,0 \%$ & - & $1,0 \%$ \\
\hline García y cols. (1995) & España & TV & $4,0 \%$ & $96,0 \%$ & - & - \\
\hline Koivula (1999) & Suecia & TV & $11,7 \%$ & $86,7 \%$ & $1,7 \%$ & - \\
\hline Duncan y cols. (2000) & EE.UU. & TV & $8,7 \%$ & $88,5 \%$ & - & $3,1 \%$ \\
\hline WACC (2000) & España & TV/Radio/ Prensa & $9 \%$ & $91 \%$ & - & - \\
\hline Ibáñez (2001) & España & Prensa & $2-4 \%$ & $96-98 \%$ & - & - \\
\hline WACC (2005) & España & TV/Radio/ Prensa & $16 \%$ & $84 \%$ & - & - \\
\hline $\begin{array}{l}\text { Frideres y cols. } \\
\text { (2006) }\end{array}$ & España & Prensa Digital & $5,8 \%$ & $91,4 \%$ & $1,6 \%$ & $1,1 \%$ \\
\hline $\begin{array}{l}\text { Frideres y cols. } \\
\text { (2006) }\end{array}$ & EE.UU. & Prensa Digital & $5,9 \%$ & $86,6 \%$ & $4 \%$ & $3,5 \%$ \\
\hline Angulo (2007) & España & $\begin{array}{l}\text { Prensa deportiva } \\
\text { (Sin fútbol) }\end{array}$ & $8,61 \%$ & $91,39 \%$ & - & - \\
\hline
\end{tabular}




\begin{tabular}{|l|l|l|c|c|c|c|}
\hline CAA (2008) & España & TV & $4,75 \%$ & $90,15 \%$ & $5,11 \%$ & - \\
\hline Ramajo y cols. 2009 & España & TV & $2,6 \%$ & $93,5 \%$ & $3,9 \%$ & - \\
\hline WACC (2010) & España & TV/Radio/ Prensa & $11 \%$ & $89 \%$ & - & - \\
\hline
\end{tabular}

Sin embargo, en la década de los noventa es cuando comienzan a disminuir las informaciones protagonizada por mujeres. En esos años las informaciones que tienen como protagonista a mujeres mantienen su frecuencia en torno al 2,45\% (en 1993 marca su frecuencia más alta un 3,27\%). Las 'mixtas', en cambio, disminuyen en esta década, de un $3,93 \%$ a un $2,87 \%$, lo que conlleva un incremento de las informaciones protagonizadas por hombres, quienes a finales de la esta década aumentan su frecuencia a un 92,5\% (a principios de los ochenta la frecuencia del hombre no llegaba al 90\%).

El siglo XXI, al contrario de lo que podría hacer pensar el hecho de que las instituciones internacionales y nacionales -el contexto político-social- han focalizado sus esfuerzos en impulsar procesos de igualdad entre hombre y mujer, se produce un retroceso en lo que a información deportiva se refiere.

Las mujeres siguen consiguiendo triunfos deportivos sin precedentes, pero siguen siendo invisibles en la prensa deportiva. Recuérdese el 13 de abril de 2003, en Londres, Paula Radcliffe consigue el récord mundial de maratón femeninas, con un tiempo de 2h.15'25", una marca que "suena" a atletismo masculino: "Radcliffe, iqué machada!", titula Marca (14-04-2003). Aun así, la noticia no es la primera de portada en ningún diario deportivo español. Sólo en el caso de Mundo Deportivo aparece en portada, con una pequeña llamada a modo de sumario, "Súper récord de Radcliffe. $2 \mathrm{~h}$. $15^{\prime} 25^{\prime \prime}$, .

Comienza el primer lustro del siglo XXI y la presencia femenina en la prensa deportiva española disminuye. Las informaciones protagonizadas por mujeres $(1,62 \%)$ pierden casi un punto en relación con el anterior período y las 'mixtas' (1,87\%) también pierden un punto, continuando la tendencia iniciada en la década anterior.

En el período comprendido entre 2005 y 2009 se inicia un leve ascenso en el que las informaciones protagonizadas por mujeres $(1,96 \%)$ y las 'mixtas' $(1,96 \%)$ recuperan unas centésimas.

Sin embargo, la realidad deportiva es otra. En los Juegos Olímpicos de Verano celebrados en Australia, Sídney 2000, de los deportistas que participaron el 38,2\% eran mujeres; en Atenas 2004 las deportistas supusieron un 40,7\% de la totalidad de los atletas; y en Pekín 2008, se vuelve a batir el récord de participación femenina, un $42,4 \%$. Los índices de las deportistas españolas son similares: un 30,50\%\% en el año 2000 ; un $41 \%$ en el 2004 ; y un $41,80 \%$ en el 2008 . Las españolas consiguieron cuatro medallas en cada una de las competiciones, el reflejo en los medios deportivos españoles, inferior al de los hombres.

5 Este record de Radcliffe será anulado por la Federación Internacional de Atletismo (IAAF) en el año 2011 debido a un cambio de normativa: "por haberlo conseguido en una carrera mixta, de participación masculina, en la que se interpreta según la nueva norma que se ayudó de "liebres" para lograrlo" (Marca, 22-09-2011). Aun así, Radcliffe sigue con el récord mundial, ya que también está en poder de la segunda marca mundial, establecida en $2 \mathrm{~h} .17$ '42" en la maratón de Londres de 2005 -la ganaba por tercera vez consecutiva-. 
En el año 2010 la frecuencia de las informaciones protagonizada por mujeres $(2,37 \%)$ y las 'mixtas' (2\%) se sitúan a niveles similares a los marcados en 1979 , es decir, al 4,37\%. Naturalmente siguen siendo los hombres los protagonistas centrales, con un 93,6\%, mejorando en un punto los datos de 1979 en detrimento de la información 'neutra' (2\%) que pierde una décima respecto del año de inicio de esta investigación.

Los datos referentes a los títulos de las informaciones dejan claro que la invisibilidad de las mujeres aumenta y su voz se apaga al analizar los títulos de las noticias en los cuales, el número de citas de personajes masculinos y nombres propios masculinos aumentan su predominio respecto de los femeninos.

En los títulos de las informaciones deportivas las citas textuales no son especialmente frecuentes. En todo caso, si se identificase la cita con la voz escuchada, serían básicamente masculinas.

Es cierto que se ha producido un aumento en los títulos de la presencia femenina a lo largo del tiempo, pero estadísticamente es insignificante, lo cual implica que la voz y presencia de los hombres van más allá del número de informaciones que protagonizan.

\section{Conclusiones}

Es preciso partir de un hecho sociológico: el deporte femenino se ha convertido en acontecimiento de las sociedades modernas, entendido como un proceso evolutivo propio de las occidentales, y que posee un trasfondo histórico que puede servir de referente, de aceptación y progreso. Se confirma la superación del paradigma de la mujer como mera receptora pasiva del fenómeno deportivo. La mujer desde finales del siglo XX y en los comienzos del siglo XXI es Deportista.

Siendo esto así, lo que se deriva de esta investigación contradice lo que podría entenderse como consecuencia lógica de ello. Y eso, porque la mujer deportista no solo no ha aumentado su presencia en las páginas de los medios deportivos, sino que la ha reducido.

- La imagen de las mujeres que la prensa deportiva española transmite está altamente desequilibrada respecto del hombre. La mujer es sujeto noticioso únicamente en el $5,11 \%$ de los casos -unas veces sola $(2,18 \%)$ y otras acompañada por al menos un protagonista masculino (2,93\%)-, mientras que el hombre lo es en el $92,24 \%$. Se mantienen los mismos porcentajes con el paso de los años.

- El predominio absoluto de los hombres va más allá del número de informaciones que protagoniza. Es también ostensible en los titulares, ya que en el 63,95\% de los títulos el nombre propio masculino es el que más abunda, mientras que el femenino solo supone el 1,99\%. También en las citas -poco frecuentes -incluidas en los títulos, son los hombres los que vuelven a estar por delante de las mujeres.

- En líneas generales la realidad mediática no refleja la realidad real. Hay un déficit claro de información deportiva cuando la mujer es protagonista. Definitivamente, no se ha producido el cambio esperado de la información sobre mujer en la prensa especializada. 


\section{Referencias bibliográficas}

ANGUERA, M ${ }^{a}$ Teresa (2011): “CĆmo se lleva a cabo un registro observacional?” Revista de Entrenamiento Deportivo, 25 (1), pp. 23-28.

ANGULO, Marta (2007): Las imágenes de las deportistas en los medios de comunicación. Madrid: Ministerio de Educación y Ciencia, Consejo Superior de Deportes.

AS. Desde 1979 - 2010.

ASOCIACIÓN PARA LA INVESTIGACIÓN DE MEDIOS DE COMUNICACIÓN [en línea]: Estudio General de Medios (EGM). http://www.aimc.es/ [consulta: 5 de marzo de 2013].

CONSEJO AUDIOVISUAL DE ANDALUCÍA(CAA) (2008): Estudio sobre género $y$ deporte en televisión. Consejo Audiovisual de Andalucía. Recurso en línea: http://www.consejoaudiovisualdeandalucia.es/sites/default/files/publicaciones/estudio_sobre_gxnero_y_deporte_en_televisixn_2008.pdf [Consulta: 22 de julio de 2013].

DUNCAN, Margaret Carlisle y MESSNER, Michael A. (1994): Gender stereotyping in televised sports: A follow up to the 1989 study. Estados Unidos: The Amateur Athletic Foundation of Los Angeles. Recurso en línea:

http://www.la84.org/gender-stereotyping-in-televised-sports-a-follow-up-to-the1989-study [Consulta: 22 de julio de 2013].

DUNCAN, Margaret Carlisle y MESSNER, Michael A. (2000): Gender in televised sports: 1989, 1993 and 1999. Estados Unidos: The Amateur Athletic Foundation of Los Angeles. Recurso en línea: http://library.la84.org/9arr/ResearchReports /tv2000.pdf [Consulta: 22 de julio de 2013].

DUNCAN, Margaret Carlisle; MESSNER, Michael A.; \& WILLIAMS, Linda (1991): Coverage of women's sports in four daily newspapers. USA: The Amateur Athletic Foundation of Los Angeles. Recurso en línea: http://www.la84.org/coverageof-womens-sports-in-four-daily-newspapers [Consulta: 22 de mayo de 2012].

DUNCAN, Margaret Carlisle; MESSNER, Michael A., WILLIAMS, Linda; \& JENSEN, Kerry. (1990): Gender stereotyping in televised sports. USA: The Amateur Athletic Foundation of Los Angeles. Recurso en línea: http://www.la84.org/genderstereotyping-in-televised-sports/ [Consulta: 22 de julio de 2013].

FRIDERES, Jill E. y PALAO, José $\mathrm{M}^{\mathrm{a}}$ (2006): “Análisis de las noticias deportivas de dos periódicos digitales de España y Estados Unidos: ¿promoción de la actividad física y el deporte?". Apunts: Educación Física y Deportes, 85, pp. 7-14.

GARCÍA, Elisabet; MORAGAS, Miquel de; y GÓMEZ, Miguel (1995): "El deporte en las televisiones españolas: Un papel creciente en la programación". Telos, 38, pp. 110-118.

HURTADO GONZÁLEZ, Silvia (2009): “Algunas peculiaridades de los titulares de actosde habla en la prensa española e hispanoamericana”. Zer, vol. 14, núm. 27, pp. 189-202 
IAAF Athletics (Web oficial de Federación Internacional de Atletismo) [en línea]: http://www.iaaf.org/. [consulta: 12 de diciembre de 2012].

IBÁÑEZ, Ma Eugenia (2001): "Información sobre deporte femenino: El gran olvido". Apunts: Educación Física y Deportes, 65, pp. 111-113.

KOIVULA, Nathalie (1999): "Gender stereotyping in televised media sport coverage”. Sex Roles, 41(7/8), pp. 589-604.

LÓPEZ HIDALGO, Antonio (2001): El titular: manual de titulación periodística. Madrid, Comunicación Social.

MARCA: desde 1979 - 2011

MUNDO DEPORTIVO: desde 1979 - 2010

NEVILL, Alan M.; LANE, Andrew M.; KILGOUR, Lindsey J.; BOWES, Neal; \& WHYTE, Gregory P. (2001): "Stability of psychometric questionnaires". Journal of Sports Science, 19, pp. 273-278

OLYMPIC (Web oficial del Comité Olímpico Internacional) [en línea]: http://www.olympic.org. [Consulta: 1 de noviembre de 2012].

RAMAJO, Natividad y LUZÓN, Virginia (2009): La representación de la mujer deportista en la televisión generalista. Un caso práctico: la televisión pública catalana (TV3 y C33). II Jornadas Mujer y Medios de Comunicación, (pp. 68-84). País Vasco: Universidad de País Vasco. Recurso en línea:

http://www.berdintasuna.ehu.es/p234-content/es/contenidos/informacion/igualdad_publicaciones/es_info/adjuntos/Jornadas_Mujer_Medios_Comunicacion_2ed. pdf [Consulta: 22 de julio de 2013].

SAINZ DE BARANDA ANDUJAR, Clara (2012): Mujeres y deporte en los medios de comunicación. Estudio de la prensa deportiva española (1979-2010). [Tesis doctoral]. Universidad Carlos III de Madrid.

SPORT: Desde 1979 - 2010

WACC (2000): Global Media Monitoring Project: Women's Participation in the News. Toronto, Media Watch Canada.

WACC (2005): Promoting Gender Equality in News Media: GMMP 2005. Toronto, Media Watch Canada.

WACC (2010): ¿Quién figura en las noticias? Proyecto de Monitoreo Global de Medios. Toronto, Asociación Mundial para la Comunicación Cristiana (WACC). Recurso en línea: http://www.adpc.cat/05 ComFem/document/Informe\%20 EspanyolGMMP_2010.pdf [Consulta: 22 de julio de 2013]. 